\title{
Scientific evaluation of an intra- curricular educational kit to foster inquiry-based learning (IBL)
}

Nathalie Debaes, Nina Cords, Amrita Prasad, Robert Fischer, Manfred Euler, et al.

Nathalie Debaes, Nina Cords, Amrita Prasad, Robert Fischer, Manfred Euler, Hugo Thienpont, "Scientific evaluation of an intra-curricular educational kit to foster inquiry-based learning (IBL)," Proc. SPIE 9289, 12th Education and Training in Optics and Photonics Conference, 92891B (17 July 2014); doi: $10.1117 / 12.2070773$

SPIE Event: 12th Education and Training in Optics and Photonics Conference, 2013, Porto, Portugal 


\title{
Scientific evaluation of an intra-curricular educational kit to foster inquiry-based learning (IBL) Nathalie Debaes ${ }^{1}$, Nina Cords $^{2}$, Amrita Prasad ${ }^{1}$, Robert Fischer ${ }^{1}$, Manfred Euler ${ }^{2}$, Hugo Thienpont ${ }^{1}$ \\ ${ }^{1}$ Vrije Universiteit Brussel, Pleinlaan 2, 1050 Brussels, Belgium; \\ ${ }^{2}$ Leibniz Institute for Science and Mathematics Education (IPN), University of Kiel, Olshausenstraße 62, 24118 Kiel, Germany.
}

\begin{abstract}
Society becomes increasingly dependent on photonics technologies; however there is an alarming lack of technological awareness among secondary school students. They associate photonics with experiments and components in the class room that seem to bear little relevance to their daily life. The Rocard Report [5] highlights the need for fostering students' scientific skills and technological awareness and identifies inquiry based learning (IBL) as a means to achieve this. Students need to actively do science rather than be silent spectators.

The 'Photonics Explorer' kit was developed as an EU funded project to equip teachers, free-of-charge, with educational material designed to excite, engage and educate European secondary school students using guided inquiry based learning techniques.

Students put together their own experiments using up-to-date versatile components, critically interpret results and relate the conclusions to relevant applications in their daily life. They work hands-on with the material, thus developing and honing their scientific and analytical skills that are otherwise latent in a typical class room situation.

A qualitative and quantitative study of the impact of the kit in the classroom was undertaken with 50 kits tested in $7 \mathrm{EU}$ countries with over 1500 students in the local language. This paper reports on the results of the EU wide field tests that show the positive impact of the kit in raising the self-efficacy, scientific skills and interest in science among students and the effectiveness of the kit in implementing IBL strategies in classrooms across EU.
\end{abstract}

Keywords: K12, education, intra-curricular, kit, hands-on, Photonics, evaluation

\section{INTRODUCTION}

The imminent lack of skilled workforce in science and technology is an increasing concern for Europe as well high-tech industries world over [2]. Research in science education has shown that the low interest in science and technology is closely related to the quality of science education and often causes young students to decide against majoring in science; especially physics [3][4]. This applies especially to girls despite the increasing amount of girls choosing to study science and technology [1]. Students seldom experience real scientific methods and inquiring activities at school, although experiments are an essential part of scientific work

On a European level, expert groups have addressed the need for renewed school pedagogy and proposed a change from the current, mostly teacher-centered environment to a more mentally activating and student oriented learning environment.

\section{INQUIRY BASED LEARNING (IBL)}

The use of inquiry-based learning (IBL) in European classrooms is recommended to achieve the goals of a knowledge based society and increase the scientific literacy of its citizens [1][5]. Besides the different role of students, IBL also includes a different teaching and classroom culture which requires a certain nature of the classroom atmosphere, the teacher, the means and the aim.

The classroom atmosphere is marked by a large amount of students' participation in class. The students and their teacher communicate dialogically and appreciate views and opinions. The teachers' role in an IBL environment is to support the involvement of their students in inquiry processes and help them with the abovementioned central parts of IBL. Instead of the more teacher-centered approach, they now play the role of a guide to their students and provide an adequate balance of challenge and learning support [6]. IBL calls for active use of hands and minds from the students and help to engage them in self-directed inquiry and, hence, in active 
learning. While the standard physics lessons focus on conveying factual knowledge, the IBL culture emphasizes more complex conceptual understanding, focusing on the development of scientific skills, social competences and open-mindedness for inquiry processes. Therefore, students develop problem solving and critical thinking and are prepared for future competitiveness and lifelong learning [7].

\section{THE PHOTONICS EXPLORER KIT}

The Photonics Explorer is an intra-curricular educational kit with didactic content based on IBL techniques. It is designed to equip teachers and encourage students to extend scientific inquiry and promote the interest of young people in science and technology. The aim of the kit is to engage, excite and educate students with physics, promote students' discovery and development of certain scientific and technical competencies, foster their openmindedness for science and improve the image of physics in school. The kit equips teachers with components for hands-on experiments, didactic framework and multimedia material. The kit addresses students in the lower and upper secondary school levels with four modules for each level.

The Photonics Explorer was developed in an EU project, with an international team of experts in photonics and didactics and teachers from more than 11 countries. The kit contains robust and versatile components for handson work in small groups of 2-3 students to ensure that a class of 25-30 students can work simultaneously in teams. The didactic content is modular in structure with 8 modules; 4 for lower secondary (12-14 years) and 4 for upper secondary (16-18 years). All modules deal with the basic, crucial aspects of light and optics such as reflection, refraction, lenses, diffraction and polarization. The didactic content is available in 8 EU languages.

The worksheets in the Photonics Explorer begin with a motivation/teaser that introduces the theme of the module to the students and raises their curiosity. The group is then asked to examine a given task and come up with their own ideas for a certain problem. At the end, the whole class discusses its findings and sometimes presents the results in small groups. For each module a fact sheet is handed out that summarizes important aspects and facts to remember for the students. Additionally, the kit also contains a teacher guide with a lesson outline and all the relevant information that the teacher needs for a certain module e.g. lesson overview, comprehensive background information and answers to questions that students might ask.

The kit is provided to teachers completely free of charge but only in conjunction with teacher training courses conducted in the local language by a professional teacher trainer. This is to ensure that the content and concepts of IBL are communicated effectively to the teachers and they can clarify any questions or doubts. Further, teachers work with the material themselves, performing the experiments and going through the didactics during the teacher training.

In order to estimate the impact of the Photonics Explorer kit on students learning, interest in science and understanding, an extensive scientific evaluation was undertaken. The quantitative evaluation was mainly conducted through specially designed questionnaires while the qualitative evaluation was based on interviews with teachers and student. The following section highlights the main research questions, evaluation process and results.

\section{SCIENTIFIC EVALUATION}

\subsection{Main research questions}

Based on the aims of the Photonics Explorer with regard to self-efficacy, interest and image of physics the following research questions were designed:

1. To what extent does interactive engagement with the Photonics Explorer promote interest and openmindedness for science and improve the image of physics?

Particularly with regard to the lack of interest among the female students and the specially designed materials for girls differences between the genders was studied with the question:

2. Are there any differences between the individual types of students or gender-specific discrepancies?

The field test and the teacher interviews provided information about the feasibility of the Photonics Explorer in class and answered the question: 
3. What is the students' and teachers' opinion of the applicability and feasibility of the Photonics Explorer in class? Does the content of the Photonics Explorer cover the curricula of the participating countries?

The description of the physics lesson by students and teachers before and after the use of the Photonics Explorer in their class clarifies the question:

4. How strong is the acceptance of using the Photonics Explorer in class and how is the implementation of IBL strategies promoted by it? What changes do teachers notice in the students and themselves?

The evaluation was done in a pre-post-follow-up design. The students were asked to fill out questionnaires

- $\quad$ right before start working with the Photonics Explorer (pre-test)

- After finishing the last module allocated to their school (post-test)

- $\quad 3$ to 4 months later (follow-up test)

\section{QUALITATIVE AND QUANTITATIVE RESULTS}

The following results are measured for 427 students of 25 classes from 7 EU countries; Belgium, Bulgaria, France, Germany, Poland, Spain and UK

\subsection{Qualitative results}

The Photonics Explorer was very well received by all teachers and students that tested the kit. In general, the worksheets and the fact sheets were found to be very good and comprehensive for the students in both lower and upper secondary levels. Most of the teachers agreed that they would make their own minor changes to the worksheets if necessary and they were encouraged to do so. The teacher guide and the integrated background information for the modules were very well accepted and showed the teachers how to use and implement each module. Even teachers with only one hour of physics lessons per week had enough time to spend on experiments.

The students had a very positive impression of the material. Both groups of younger (12-14 years) and older (16-18 years) students liked working in small groups and hands-on with sufficient material for each student. Especially modern tools like the lasers were extremely appreciated by the older students. All students were engaged in doing experiments and more actively involved in the lesson and some teachers got the impression that their students had a better theoretical understanding.

\subsection{Quantitative results}

Implementation of the Photonics Explorer in the classroom showed significant positive short term effects in terms of the improvement of self-efficacy and interest of students overall. The initial amount of IBL in the classroom directly influenced both the initial self-efficacy and the interest of students and also the image of physics the students already had. The more IBL techniques the teacher used in the classroom, the higher interest and self-efficacy of the students and the better their image of physics. The initial amount of IBL the students had earlier in class is one of the bases for the interest in physics as a subject. The more IBL techniques the teachers used, the higher the initial mean of the interest before starting with the Photonics Explorer kit. In all groups the interest in physics increased after working with the material but only significantly for the students with small and medium amount of initial IBL methods. The highest gain was found in classes with medium amount of IBL. 


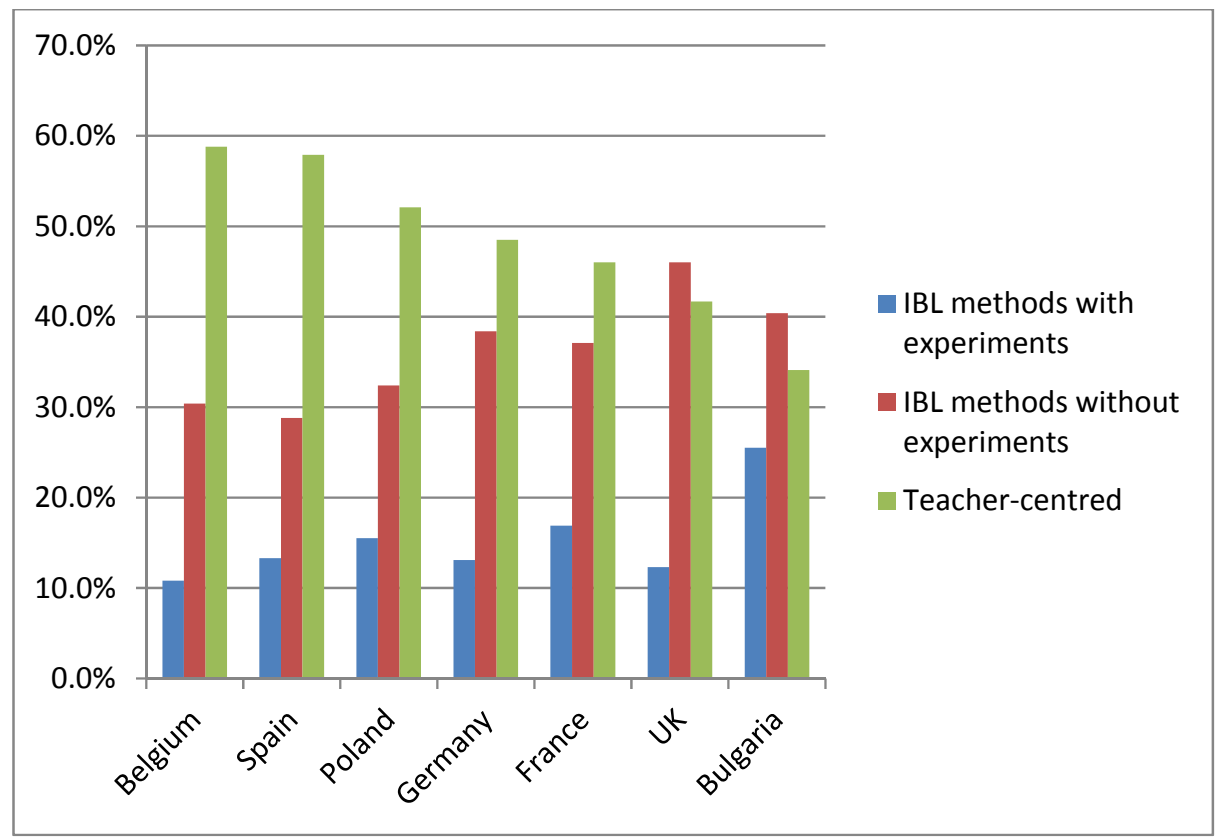

Figure 1: IBL methods in the test countries

The separation by gender of the students revealed relatively similar initial values. The boys were more interested in physics lessons after working with the Photonics Explorer. For girls, their self-efficacy increased after using the kit.

\section{CONCLUSION}

The evaluation study was designed to evaluate the qualitative and quantitative impact of the Photonics Explorer kit in the classroom. To this end, four significant research questions were designed and the study aimed to answer these questions based on data collected during the field test phase of the PE.

The impact evaluation of the Photonics Explorer clearly showed the positive role of Inquiry Based Learning, especially in those classrooms where the initial amount of IBL was medium to low. The results of the study showed a significant increase in students' self-efficacy after working with the kit in physics lessons. Especially students with a rather small or medium amount of initial IBL profited from the intervention. The Photonics Explorer worked especially well with lower secondary students.

For the male students, their interest in physics rises after working with the PE while girls feel more confident in their scientific ability and their self-efficacy rises.

\section{REFERENCES}

[1] Commission of the European Communities (2009). Communication from the Commission to the European Parliament, the Council, the European Economic and Social Committee of the Regions.

[2] OECD (2006). Evolution of student interest in science and technology studies. Policy report. Paris: OECD.

[3] Osborne, J., \& Collins, S. (2001). Pupils' views of the role and value of the science curriculum: A focus-group study. International Journal of Science Education, 23 (5), 441-67.

[4] OECD. (2009a) Technical Report- PISA 2006. Paris: OECD Publishing

[5] European Commission (2007). Science education now: A renewed pedagogy for the future of Europe. Luxembourg: Office for Official Publications of the European Communities.

[6] Chin, C. (2007). Teacher Questioning in Science Classrooms: Approaches that Stimulate Productive Thinking. Journal of Research in Science Teaching, 44(6), 815-843.

[7] Rennie, L. (2007), Learning science outside of school, In: Handbook of Research on Science Education, Ed. S K Abell and N G Lerderman (New Jersey: Lawrence Erlbaum Associates), 125-67 